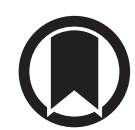

CrossMark

\title{
Host-microbe cross-talk in the lung microenvironment: implications for understanding and treating chronic lung disease
}

\author{
Reinoud Gosens ${ }^{1}$, Pieter S. Hiemstra (10 ${ }^{2}$, Ian M. Adcock (10 ${ }^{3}$, Ken R. Bracke ${ }^{4}$, \\ Robert P. Dickson (10 5,6 Philip M. Hansbro ${ }^{7,8}$, Susanne Krauss-Etschmann ${ }^{9,10}$, \\ Hermelijn H. Smits (1) ${ }^{11}$, Frank R.M. Stassen ${ }^{12}$ and Sabine Bartel (10,13
}

\begin{abstract}
Affiliations: 'University of Groningen, Dept of Molecular Pharmacology, GRIAC Research Institute, Groningen, The Netherlands. ${ }^{2}$ Dept of Pulmonology, Leiden University Medical Center (LUMC), Leiden, The Netherlands. ${ }^{3}$ Airways Disease, National Heart and Lung Institute, Imperial College London, London, UK. ${ }^{4}$ Dept of Respiratory Medicine, Ghent University Hospital, Ghent, Belgium. ${ }^{5}$ Division of Pulmonary and Critical Care Medicine, Dept of Internal Medicine, University of Michigan Medical School, Ann Arbor, MI, USA. ${ }^{6}$ Michigan Center for Integrative Research in Critical Care, Ann Arbor, MI, USA. ${ }^{7}$ Priority Research Centre for Healthy Lungs, Hunter Medical Research Institute and the University of Newcastle, Newcastle, Australia. ${ }^{8}$ Centre for Inflammation, Centenary Institute and University of Technology Sydney, Faculty of Science, Sydney, Australia. ${ }^{9}$ Early Life Origins of Chronic Lung Disease, Research Center Borstel, Leibniz Lung Center, Airway Research Center North, Member of the German Center for Lung Research (DZL), Borstel, Germany. ${ }^{10}$ Institute for Experimental Medicine, Christian-Albrechts-Universitaet zu Kiel, Kiel, Germany. ${ }^{11}$ Dept of Parasitology, Leiden University Medical Center, Leiden, The Netherlands. ${ }^{12}$ Dept of Medical Microbiology, NUTRIM - School of Nutrition and Translational Research in Metabolism, Maastricht University Medical Center, Maastricht, The Netherlands. ${ }^{13}$ University of Groningen, University Medical Center Groningen, Dept of Pathology and Medical Biology, GRIAC Research Institute, Groningen, The Netherlands.
\end{abstract}

Correspondence: Sabine Bartel, University Medical Center Groningen, Hanzeplein 1, 9700 RB Groningen, The Netherlands. E-mail: s.r.barteldumcg.nl

@ERSpublications

The reciprocal interaction between microbes and host in the lung is increasingly recognised as an important determinant of health. The complexity of this cross-talk needs to be taken into account when studying diseases and developing future new therapies. https://bit.ly/2VKYUfT

Cite this article as: Gosens R, Hiemstra PS, Adcock IM, et al. Host-microbe cross-talk in the lung microenvironment: implications for understanding and treating chronic lung disease. Eur Respir J 2020; 56 : 1902320 [https://doi.org/10.1183/13993003.02320-2019].

ABSTRACT Chronic respiratory diseases are highly prevalent worldwide and will continue to rise in the foreseeable future. Despite intensive efforts over recent decades, the development of novel and effective therapeutic approaches has been slow. However, there is new and increasing evidence that communities of micro-organisms in our body, the human microbiome, are crucially involved in the development and progression of chronic respiratory diseases. Understanding the detailed mechanisms underlying this crosstalk between host and microbiota is critical for development of microbiome- or host-targeted therapeutics and prevention strategies. Here we review and discuss the most recent knowledge on the continuous reciprocal interaction between the host and microbes in health and respiratory disease. Furthermore, we highlight promising developments in microbiome-based therapies and discuss the need to employ more holistic approaches of restoring both the pulmonary niche and the microbial community.

Received: 30 Nov 2018 | Accepted after revision: 20 April 2020

Copyright OERS 2020. This version is distributed under the terms of the Creative Commons Attribution NonCommercial Licence 4.0. 


\section{Introduction}

In the past decade, we have learned that the lungs, previously considered sterile, in fact harbour a dynamic ecosystem of diverse bacteria, fungi and viruses [1]. This lung microbiota is detectable in health [2, 3], altered in disease [4], predictive of disease outcomes [5, 6] and correlates with variations in host immunity $[7,8]$. Recent insights, based on studies in both humans [8] and mice [7], are that the baseline immune tone of the lungs, even in health, is closely linked to the local microbial milieu [9]. This hypothesis that the "immune tone" in the airways and alveoli is at least in part regulated by the microbiota is a radical departure from our conventional, dichotomous understanding of lung immunity: dormant in health, activated in infection. Next to known changes in the inflammatory milieu of the lungs, most lung diseases, such as asthma [10], COPD [11], cystic fibrosis [12], idiopathic pulmonary fibrosis (IPF) [13] and, recently, lung cancer [14] have been associated with a microbial dysbiosis in the lung. However, it is unknown if changes in the bacterial composition drive disease pathogenesis or if they are rather a reflection of alterations of the ecological niche $[9,15]$. Thus, it is of utmost importance to understand the underlying molecular mechanisms at the host-microbiome interface to develop novel targeted therapies or preventative approaches.

Here, we discuss the impact of host-microbiome cross-talk on respiratory health and disease, while focusing on how the local microbiota and the host interact at the epithelial surface. Furthermore, we review current therapeutic approaches, and suggest a more holistic approach for treating lung disease in the future. This review is a follow-up of presentations and discussions at and after the ERS Research Seminar "Cross-talk in the lung microenvironment: implications for understanding chronic lung disease" (Berlin, February 2018).

\section{The mucosal niche in the lung}

All external interfaces of the human body, such as gut, skin, reproductive and respiratory tracts, are colonised by a distinct microbial flora [16]. The microbial communities differ at the various body sites due to local factors (e.g. oxygen, carbon dioxide, $\mathrm{pH}$, nutrients, host defence factors, inhaled pollutants) that shape the niche. As examples, the lumen of the lower gastrointestinal tract represents a low-oxygen, nutrient-rich environment and is thus commonly populated by high-abundance communities of anaerobic organisms; by contrast, the skin is a low-nutrient environment directly exposed to environmental oxygen and is thus more commonly populated by relatively sparse communities of oxygen-tolerant bacteria. Thus, the local environment is a crucial determinant of the formation of microbial communities early in development, but also at later stages [17].

Consequently, the lung microenvironment creates a special ecological niche for microbes that differs from other body sites and will presumably influence niche-specific colonisation. Accordingly, airway epithelial cells are a principle contributor in shaping this niche, as they are strategically located to be the first contact between inhaled substances (including micro-organisms) and host tissues. Various mechanisms are employed by the airway epithelium in host defences against infection, including its barrier function, mucociliary clearance, production of antimicrobial peptides and other substances, pro- and anti-inflammatory mediators and ability to transport (e.g. polymeric IgA and IgM from the basal to the apical side of the epithelium through the polymeric immunoglobulin receptor (pIgR)) [18-21]. It is likely that similar mechanisms contribute to the formation of niche-specific communities along the respiratory tract, and such local conditions are crucial for allowing beneficial microbiota to persist at epithelial surfaces. The involvement of such host-microbiota interactions in disease is well illustrated in cystic fibrosis. In cystic fibrosis, mutational dysfunction of the cystic fibrosis transmembrane regulator (CFTR) protein results in a reduction of anion (mostly bicarbonate and chloride) exchange across the epithelial surface, resulting in a dehydrated surface and sticky mucus. This mucus cannot be readily cleared from the airways, which helps to explain why CFTR mutations are associated with alterations of the resident microbiota, including frequent colonisation with Staphylococcus aureus and Pseudomonas aeruginosa (as recently reviewed in [22]). However, we are still lacking knowledge on the underlying mechanisms of the niche alterations in more complex genetic lung diseases, such as asthma and COPD.

Additionally, the epithelium transmits environmental and microbial signals to the immune system, allowing it to mount appropriate responses or develop tolerance [21, 23, 24].

Various mechanisms of sensing microbial presence include pattern recognition receptors such as the Toll-like receptors [20], and other processes not mediated via classical receptor-mediated signalling, e.g. the integrated stress response [25]. Upon sensing microbial, environmental or endogenous challenges, epithelial cells mount active responses by increasing defence molecules, such as antimicrobial peptides, cytokines and chemokines. These enhance the defence against respiratory pathogens, while simultaneously changing the ecological niche of complex microbial communities, indicating that the properties of the ecological niche encountered by micro-organisms changes as a result of environmental exposures. 
Anatomical differences along the respiratory tract such as differences in epithelial cell composition shape this ecological niche. While the epithelium contributes to local conditions that shape the microbiota, epithelial exposure to microbes and their products has marked effects on its function. Consequently, human epithelia and the microbiota have developed interactions that are of mutual benefit, responding to pathogens and tolerating innocuous substances [26]. This concept is important to understand how inhaled environmental triggers regulate immunity, since microbial components contribute to the response to environmental exposures, such as farm [27] and geogenic (earth-derived) dust [28]. However, how the epithelium integrates these microbial and nonmicrobial signals into a fine-tuned response is incompletely understood.

In order to transmit signals from the environment, epithelial cells use sophisticated communication systems with other lung cell types. The airway epithelium (and that of gut and skin) plays key roles in transmitting signals from micro-organisms and environmental stimuli to instruct antigen-presenting dendritic cells to direct immunity towards inflammation or tolerance [24]. Epithelial cells interact not only with other immune cells, such as macrophages, neutrophils, innate lymphoid cells and T-cells, but also with structural cells such as fibroblasts, airway smooth muscle cells and endothelial cells via a plethora of different mechanisms (figure 1) [18, 20, 24]. This array of interactions with environmental triggers, micro-organisms and lung cells allows epithelial cells to orchestrate host defence and immunity, and to maintain epithelial integrity and mediate pathologic airway remodelling (figure 1) [21].

\section{Establishment and maintenance of the lung microbiota}

The establishment of the lung microbiota likely occurs in the first days and weeks of life. Although early high-profile reports suggested the presence of a "placental microbiome" that could influence prepartum lung development [29], subsequent well-controlled studies have failed to detect bacterial signals distinct from contaminating DNA present in negative controls [30]. The lung microbiota of newborn mice is below the limit of detection (via quantitative PCR), and increase in total burden in the following days and weeks $[31,32]$. In human infants, the composition of the respiratory microbiome seems to mature in a predictable, well-characterised pattern during the first year of life [32]. Besides the special niche characteristics of the lung, early-life respiratory microbiota are influenced by mode of delivery (vaginal versus caesarean), method of feeding (breast versus bottle), and exposures (siblings, day-care attendance) [32]. Provocatively, early-life respiratory microbiota may predict subsequent susceptibility to respiratory tract infections $[32,33]$, suggesting roles of the local microbes in immune homeostasis and resistance to pathogens. In contrast, being exposed to an increased microbial diversity during childhood promotes the development of balanced immunity and is protective against inflammatory responses to allergens and asthma development $[34,35]$. This protection is partly associated with distinct farm dust, which in vitro increases epithelial barrier function and antiviral defences [27].

Once established, the composition of the lung microbiome is determined by three ecological factors: immigration, elimination and relative growth rates of community members (figure 1) [36]. In health, lung communities are sparse and dynamic, largely determined by the equilibrium between immigration (via microaspiration [3]) and elimination (via cough, mucociliary clearance and immune defences). Little evidence supports the presence of resident lung bacteria in health that reproduce and survive selective pressures $[1,4,9]$. Nevertheless, the transient and dynamic communities detected in health are largely viable [37], and exert a detectable effect on the immune constitution of the lower respiratory tract $[7,8]$.

The establishment of a diverse respiratory microbial flora is influenced by many different factors. There is a fine-tuned balance between tolerance of commensal or "beneficial" bacteria at the epithelial surface, and the development of active immune responses to pathogens. Notably, this balance is regulated by both hostand microbe-derived signals. Sudden shifts in this tightly controlled equilibrium, such as outgrowth of specific pathogens or, for example, virally induced damage to the airway epithelium destroying the barrier and inducing local immune responses, can have detrimental effects on both the host and the microbiome, and might therefore contribute to the pathogenesis of respiratory diseases.

\section{Role of the environment in shaping the lung microbiome}

Environmental influences play pivotal roles in the development of lung diseases. This might be due to direct effects on the host epithelial barrier and immune responses, but most known risk factors, such as cigarette smoke, air pollution [38, 39], viral infections and diet also directly impact the microbiota [11, 40-42]. Thus, a combination of both might be critical for disease development.

For example, one of the most studied inhaled toxins, cigarette smoke, supposedly has a detrimental effect on both the host and microbial communities. Prolonged cigarette smoke exposure contributes to increased baseline inflammation in the airways and epithelial remodelling towards goblet cell hyperplasia and a reduction in cilia and ciliary activity [19] and can reduce the antimicrobial defence provided by the airway 


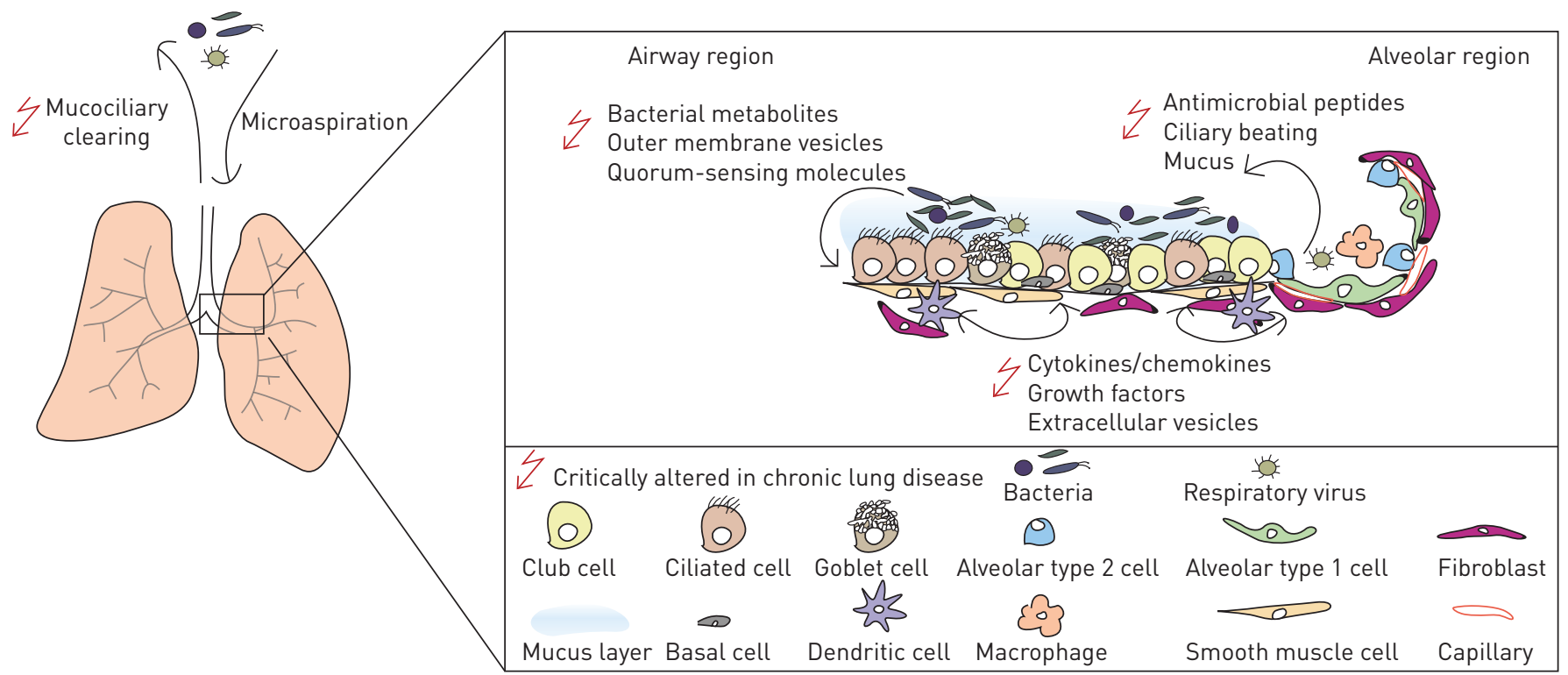

FIGURE 1 Host-microbiome cross-talk in the lung microenvironment. The lung microenvironment consists of different cell types, depending on the location in the proximal-to-distal airway tree. The epithelial layer in larger airways is constantly exposed to a variety of different microbes of the local microbiota. While the composition of the latter is influenced by host factors such as elimination via mucociliary clearance, it also depends on the competition among the microbial inhabitants. It is now evident that there is a complex cross-talk between host and microbes in this environment. Accordingly, bacterial metabolites or outer membrane vesicles can influence the host status, while antimicrobial peptides or cytokines can shape the composition of the microbiota. As virtually all of these single factors are altered in chronic respiratory disease, it is of utmost importance to appreciate and assess the complexity of this system in future studies.

epithelium [43]. In patients with COPD, it has been shown to be associated with reduced lung Bacilli and increased Haemophilus influenzae and Streptococcus pneumoniae [44], but the largest study to date in "healthy" smokers has not found a significant effect of cigarette smoke on the lung microbiome [45]. Thus, it is intriguing to speculate that cigarette smoke primarily affects the host system, which over time and upon disease (COPD) development in susceptible individuals may also affect the microbiome.

Another example of environmental influence on respiratory disease is diet, which has profound effects on both microbiota and health, even in the short term [46]. The intake of dietary fibre induces similar beneficial microbiota changes in the lung and gut [47]. While low-fibre diets change the gut microbial community over multiple generations in mice [48], high-fibre diets have beneficial effects in pregnant mice and suppress allergic airway disease (AAD) in mothers and their offspring [49]. This also highlights the importance of cross-talk between the gut and lung. High-fibre diets induce the production of short-chain fatty acids by gut bacteria, which are transported systemically to the lung, where they exert anti-inflammatory actions and ameliorate $\mathrm{AAD}$ in mice [47]. In contrast, a lipid-rich diet alters the microbiota and promotes metabolic inflammation [50], and has been associated with premetastatic niche development in lung cancer [51]. However, the detailed mechanisms of action remain unclear.

Along with bacteria, common major respiratory viruses, including respiratory syncytial virus, rhinovirus, coronaviruses, influenza and adenoviruses are part of the respiratory microbiome [52] and contribute to the pathogenesis of chronic respiratory diseases. This may result from complex interactions of viruses with the host's immune system and the microbiota including other pathogens. These interactions can influence the prevalence of bacterial pathogens by increasing the expression of adhesion molecules on the respiratory epithelium, damaging respiratory epithelial cells compromising barrier function, impairing mucociliary clearance and altering host immunity and the lung microenvironment [53-55]. Interestingly, it has been suggested that viruses (and bacteriophages) induce consistent and reproducible changes in respiratory microbiomes in chronic disease, but not the healthy state, and have been shown to increase microbial diversity in the nasopharynx $[53,56,57]$. Furthermore, fungi such as Aspergillus spp. contribute to the pathogenesis of chronic respiratory diseases as pathogens on their own, but also by activation of the immune system, and probably through their interactions with microbiota, particularly with nontuberculous mycobacteria $[9,58]$.

Importantly, the absence of certain members from the microbiome can have detrimental influences on lung health. This is illustrated by the relative disappearance of parasitic worms, which have been a constant part of our gut microbiome and have even been found in fossils from the period of jawed fish [59]. In fact, our 
"modern" immune system developed in the continuous presence of helminths [60], which may explain their important regulatory role in immunity. Typically, worms induce type- 2 immune responses which are considered instrumental in host defence against these parasitic infections. However, worm infections induce regulatory responses that are aimed to suppress immune responses directed at worm antigens, which allows worms to live in their host for years. Additionally, this regulatory response has a bystander effect by promoting allergen tolerance [61]. This is illustrated by studies in mouse models, in which various helminth infections have been shown to provide protection against the development of allergic asthma [62]. The range of helminth-induced protective mechanisms includes the inhibition of interleukin (IL)-33 [63] in the airways, and the induction of regulatory T-cells [64, 65], B-cells [66, 67] and macrophages [68]. Intriguingly, the presence of helminths also affects the composition of the bacterial community, which might be important for the protection against allergic airway disease in mice [69].

\section{Niche and microbiome alterations in respiratory disease}

In disease, the ecology of the lower respiratory tract changes dramatically. The airways and alveoli, normally inhospitable to bacterial reproduction [3], are radically altered by the influx of nutrient-rich oedema and mucus, establishment of stark oxygen gradients, surge of bacterial growth-promoting inflammatory responses $[70,71]$ and impairment of local host defences [36, 72]. Thus, it is unsurprising that cross-sectional studies have identified altered lung microbiomes in established lung diseases, as changes in ecological niches supposedly result in different microbial communities due to selective pressure. The composition of the lung microbiome then becomes increasingly determined by the relative growth rates of its constituents.

While there are some studies on the role of the microbiome in IPF [13] and lung cancer [14], most knowledge is based on studies of chronic inflammatory respiratory diseases, such as asthma, COPD and cystic fibrosis. Generally, those patients have increased susceptibility to infections and exacerbations that again affect the microbiome $[4,73,74]$. H. influenzae, Moraxella catarrhalis and S. pneumoniae are associated with the development of (severe) asthma and COPD. They modify the lung microbiome and drive inflammation, oxidative stress, symptoms and exacerbations [44, 75-80], which may form a vicious circle perpetuating the disease. $M$. catarrhalis and $H$. influenzae in particular induce neutrophilic inflammation, more severe disease, and steroid resistance [79-84]. Furthermore, in asthma, altered respiratory microbiome profiles are associated with asthma phenotype [85] and severity [86], responses to allergens [87] and treatment [88].

In cystic fibrosis and bronchiectasis, Haemophilus and Pseudomonas spp. increasingly dominate the lung microbiota [89, 90]. Genetic association studies in Phe508del cystic fibrosis-affected homozygous individuals have shown associations of single nucleotide polymorphisms with important disease features related to bacterial colonisation. For example, gene variants of human leukocyte antigen class II genes (implicating a role for $\mathrm{T}$-cell and B-cell immunity) associate with age of onset of persistent P. aeruginosa infection, indicating the role of the host's immune system in selecting a distinct microbiota [91].

Like the bacterial composition of the lung microbiome, antiviral responses are altered in chronic respiratory diseases. In asthma, where airway epithelial repair in response to viral infection is aberrant due to changes in genes regulating epithelial barrier function and repair [92], the airway epithelium has reduced innate immunity to common viruses such as rhinovirus $[79,93]$. This is due to reduced type I and III interferon and increased miRNA levels [94, 95]. Transforming growth factor (TGF)- $\beta$, a cytokine commonly upregulated in asthma, suppresses airway epithelial innate immune responses through suppressor of cytokine signalling (SOCS)-1 and SOCS-3, contributing to impaired antiviral immunity [96]. This impaired antiviral immunity may also alter the bacterial microbiome and contribute to co-infections by bacteria [97]. Rhinovirus infection can also impair the phagocytosis of bacteria by alveolar macrophages which can lead to bacterial outgrowth in COPD [98]. Murine studies showed that influenza-infected epithelial progenitors of the distal lung exhibited severely impaired renewal capacity due to virus-induced blockade of $\beta$-catenin-dependent Fgfr2b signalling [99]. This could contribute to impaired repair of the distal lung in COPD, although its involvement in human disease is still unclear. Thus, viral and bacterial infections, and the inability to resolve them, may aggravate impaired airway and alveolar repair. Combined with a heightened airway and/or alveolar immune tone, aberrant repair mechanisms following pathogen exposures in vulnerable individuals might represent a mechanism for how host-microbiome interactions contribute to disease progression. It is currently unclear if alterations in the lung microbiome can also precede lung structural changes and inflammation and thereby contribute to onset of disease.

As discussed earlier, the relationship between lung dysbiosis and lung inflammation is bidirectional: disordered lung communities trigger epithelial and luminal inflammation, further altering growth conditions of the lung microenvironment, chronic inflammation and perpetuating dysbiosis $[4,36]$. A common 
finding across inflammatory lung conditions is the outgrowth of the inflammation-associated Proteobacteria phylum [4], and in mice the lung microbiome has been shown to be associated with pulmonary levels of the innate cytokine IL-1 $\alpha$ [7]. Additionally, alterations in the microbiome of the gut might influence the immune tone of the lung, which is shown by the development of more severe experimental asthma in germ-free mice [31, 47] or mice with disturbed microbiomes due to early-life antibiotic treatment $[100,101]$. In contrast, the absence of microbiota in germ-free mice or due to antibiotic treatment has recently been shown to protect mice with Kras mutations and p53 loss from lung cancer development [102]. According to the authors, this effect was due to the induction of IL-1 $\beta$ and IL-23 through the microbiota that led to IL-17 production by $\gamma \delta$-T cells and tumorigenesis.

In humans, COPD patients are two to three times more likely to have Crohn's colitis, and 50\% of people with inflammatory bowel disease also have pulmonary inflammation [103-106]. The gastrointestinal tract has by far the highest microbial content, and thus an interaction of gut microbiota and their metabolites with the gastric mucosa affects systemic immunity, which may in turn impact the lung [107]. Consequently, alterations in gut microbiota and physiology might contribute to respiratory disease and vice versa, possibly via the gut-lung axis. However, it is important to note that germ-free breeding, and to some extent antibiotic treatment, will affect both the gut and the lung microbiome, so it is still not completely clear whether these effects can fully be explained by gut-lung cross-talk or if they are influenced by aberrant local microbiota-immune cross-talk. Along this line, in mice the lung immune tone has been suggested to be more correlated to lung bacteria than gut bacteria [7].

In contrast, in mice, colitis-induced pulmonary pathology was associated with increased inflammation and Gram-negative bacterial endotoxins in the lung, that probably emanate from the gut [104]. Furthermore, cigarette smoke-induced experimental COPD models [108-110] indicate that reduced gas exchange and hypoxia in the lung is associated with hypoxia in the gut, causing colon remodelling, cell death, inflammation and impaired barrier function [111]. Additionally, activation of NOD-like receptors by bacteria in the gut increase production of reactive oxygen species by alveolar macrophages, suggesting that the gastrointestinal microbiome contributes to oxidative stress in the lung [112]. Conversely, oral application of beneficial probiotic bacteria (Bifidobacterium- and Lactobacillus-based) reduced airway inflammation and emphysema in cigarette smoke-exposed mice [113].

A large Canadian study reported that four bacterial genera Lachnospira, Veillonella, Faecalibacterium and Rothia were reduced in the faeces of human infants that subsequently developed asthma, and inoculating a human faecal microbiome supplemented with these four taxa into germ-free mice partially protected their F1 progeny from experimentally induced AAD [114]. Thus, there are encouraging studies in mouse models highlighting cause and effect of the gut-lung axis in respiratory disease. However, findings first need to be validated in humans before proposing this cross-talk as a treatable trait for respiratory disease.

In summary, at the host-microbiome interface, disordered communities are probably both cause and effects of host inflammation. However, given the close reciprocal interactions between the microbiome and host at all times, it might be impossible to determine the real cause of the very first changes in disease development, as host and microbiome factors are coinciding and closely intertwined.

\section{Implications for the development of novel therapies}

Due to the strong and dynamic interdependency between host and microbiome in local niches, it is unsurprising that most drugs used in clinical practice that were designed to target the host also affect the microbiome. Accordingly, inhaled corticosteroids and proton pump inhibitors affect the lung microbiota [115-117], as well as subsequent pneumonia risk [118, 119]. Macrolide antibiotics, broadly effective across chronic lung conditions such as COPD [120], affect both lung microbiota and host immunity. Perhaps most provocatively, baseline differences in lung microbiota appear to predict patient responsiveness to therapies such as inhaled corticosteroids [10], suggesting that variation in lung microbiota may represent an untapped phenotype of "precision medicine" in the lung. This opens new possibilities to exploit this important cross-talk in therapeutic interventions, but in order to do so, we first need to improve our understanding of the molecular mechanisms.

Increasing evidence of the importance of the microbiome raises the concept of restoring "diseased" microbiomes to prevent or treat diseases using microbiota-directed therapies or host-targeted therapies, such as probiotics, metabolites, lung microbiota transplantation or vitamin D therapy, which we discuss here in more detail.

Many clinical studies have investigated the efficacy of probiotic bacteria, which are supposedly beneficial for the host, to prevent chronic diseases such as asthma or allergic rhinitis. However, these studies have largely produced contradictory outcomes [121], which might be due to the fact that the used probiotics were not selected based on potential mechanistic effects and may not be ideal. The microbiome is a 
complex ecosystem comprised of a variety of different inhabitants and influenced by many external (host-derived) factors; thus, the addition of single strains may not make a profound difference. Thus, the transfer of whole microbiomes via faecal microbiota transplantation (FMT) could be a more promising approach. The introduction of healthy microbiota into diseased hosts has restored immunity and physiology [122], demonstrating that intestinal microbiota and their products can modulate host immunity locally and systemically, and that FMT can replace disease-related microbiomes with healthy ones [40, 107]. FMT is remarkably (90\%) successful in treating antibiotic-resistant Clostridium difficile-induced colitis [123], and is now being used as treatment in selected patients [124, 125]. The new microbiome engrafts quickly and lasts for at least a month, indicating a potential difficulty in inducing long-term beneficial changes in the microbiome via only targeting the microbial side. While there are encouraging data, questions remain whether FMT may also affect lung health, and whether lung microbiota transplantation is feasible. Furthermore, recently, severe complications of FMT have been reported due to transfer of drug-resistant bacteria [126]. Thus, it is essential to determine whether such approaches, that so far only transiently change the microbiome, can be used for the required long-term treatments of chronic (lung) diseases that coincide with a variety of structural changes, aberrant mucociliary clearance, and many more [40].

Along with living bacteria, specific microbial molecules such as lipopolysaccharide (LPS) and peptidoglycan can induce or modulate inflammatory responses [127-129]. In addition, culture supernatants of probiotic bacteria display anti-inflammatory effects, which have been ascribed to the presence of secreted immune-modulatory metabolites [130]. For example, culture supernatants of certain probiotic Bifidobacterium species decreased the secretion of type 2 cytokines from immune cell lines and the expression of costimulatory molecules on primary dendritic cells [131]. A likely mechanism is quorum sensing. Quorum sensing is a means of communication among bacteria of the same species to coordinate effector functions such as biofilm formation, sporulation or toxin secretion. The best-described quorum sensing molecules are acyl homoserine lactones (AHLs) [132]. Several AHLs are targeted by the host to interfere with growth of pathogens [133], and are in turn exploited by bacteria to regulate host gene expression for their benefit. Some AHLs can bind to distinct bitter taste receptors expressed on the airway epithelium [134] and innate and adaptive immune cells $[135,136]$, thereby modulating barrier and immune functions. The most-studied AHL, 3-O-C12-HSL, can activate phagocytes to increase phagocytosis, expression of adhesion receptors and chemotaxis [137, 138], but is itself cleaved and inactivated by airway epithelial cells [133].

Another example of bacterial-derived modulators of the host's immune responses are outer membrane vesicles (OMVs) [139]. OMVs are spherical bilayered membrane vesicles released from the surface of both Gram-negative and Gram-positive bacteria, and contain much of the biological material from the parent bacterium, but in a nonreplicative form [140,141]. Evidence suggests that the release of OMVs provides bacteria with competitive advantages when exposed to acute and chronic host-associated stressors [142]. They may protect bacteria against innate and adaptive immune responses [143-145], antimicrobial peptides and antibiotics [146, 147]. Moreover, OMVs contain factors (e.g. siderophores) aiding in the acquisition of nutrients in an environment devoid of crucial elements such as iron [147]. Besides supporting the survival of the parent bacteria, OMVs may also play role in the progression of pulmonary diseases. Bacteria frequently associated with COPD exacerbations are known to release OMVs [148]. Furthermore, macrophages stimulated with OMVs derived from prominent airway pathogens such as $P$. aeruginosa, $H$. influenzae or $M$. catarrhalis release higher amounts of tumour necrosis factor- $\alpha$ and IL-6 [148]. Legionella-derived OMVs significantly enhanced bacterial replication in macrophages [149], and bacteria-free $P$. aeruginosa OMVs have been shown to potently induce pulmonary inflammation in mice [150], strengthening the idea that OMVs exert disease-promoting activities. In addition, OMVs have been shown to induce tolerance and hyporesponsiveness, thereby facilitating bacterial adherence to and internalisation by macrophages, which may contribute to clearance of the infection [149, 151]. Thus, despite our increasing knowledge on OMVs and their potential role in interkingdom communication, there is a need for further research to better understand their pathogenic properties and possible therapeutic or prophylactic implications (e.g. novel vaccines).

An interesting alternative to FMT or probiotic bacteria might be to use immune-modulatory microbial metabolites or "beneficial" OMVs. Such chemically defined bacterial substances could be produced at large scale under controlled conditions, applied in defined (effective) doses both systemically or locally, and may have fewer adverse side-effects, i.e. in immunocompromised patients, compared to live bacteria [152]. Several studies have already used defined bacterial metabolites to treat AAD in mice. LPS from Escherichia coli O111 [153], bacterial polysaccharide A [154], oligodeoxynucleotides with bacterial CpG motifs [155], flagellin B [156], short-chain fatty acids [47], D-tryptophan [157] and the neutrophil-activating protein from Helicobacter pylori [158] all suppressed airway eosinophilia and type 2 T-helper cell-mediated 
immune responses in murine models of AAD. Likewise, several molecules from the parasite helminths have now been identified, among which excretory-secretory (ES)-62 and cystatin (AvCystatin) from Acanthocheilonema vitae, TGF- $\beta$ mimic (TGM) and Heligmosomoides polygyrus alarmin release inhibitor (HpARI) from $H$. polygyrus and IL-4-inducing principle from schistosome eggs (IPSE) are the most promising, with effects in allergic asthma models [159-163].

Nevertheless, there are significant limitations concerning the use of bacterial or parasite metabolites for therapeutic or preventative approaches. These molecules have a very narrow window of beneficial and therapeutic doses, which might be difficult to control in local tissues. Furthermore, the detailed mechanisms of action remain largely unknown, hampering translation to human studies. And finally, single metabolites, just as single bacterial strains, might not be sufficiently effective to treat or prevent complex chronic diseases with highly aberrant gut and lung microenvironments. Thus, further studies are urgently needed to determine the mode of action, but also the efficacy of single or combined metabolites to develop novel metabolite-based treatment options.

\section{Combining host and microbiome targeted therapeutic approaches}

While microbiome-based therapies are intriguing, it is important to restore the ecological niche in the lung. A diseased lung forms a completely different habitat for microbes favouring a different microbial flora. In cystic fibrosis and bronchiectasis Haemophilus and Pseudomonas spp. increasingly dominate the lung microbiota, while specific antibiotic treatment further promotes their outgrowth [89, 90]. This could be a consequence of reduced competition from commensals being destroyed by the antibiotics, or the persistent aberrant ecological niche in cystic fibrosis lungs. Supporting the latter, a recent study showed that the use of ivacaftor to increase CFTR function in patients with the G511D mutation, thereby partially restoring mucociliary clearance, rapidly decreased P. aeruginosa burden in sputum of patients [164]. However, $P$. aeruginosa was not eradicated, and outgrowth of $P$. aeruginosa was observed after 1 year of treatment, which might be due to incomplete restoration of the niche [164].

Thus, it seems inevitable that in order to restore the shifted host-microbe balance in chronic lung diseases, it is necessary to restore epithelial barrier and repair function. Failure to achieve this may result in recurrence of microbial dysbiosis. Restoration of epithelial cell function could be achieved by inducing expression of antimicrobial peptides through vitamin D treatment (which also has anti-inflammatory effects) or by restoring the barrier and antiviral immunity by farm-dust treatment [27].

\section{Conclusions and knowledge gaps}

There is substantial evidence for the functional involvement of the microbiome in chronic respiratory diseases, making it an interesting target for future therapies (figure 2). Targeting the microbiome via FMT

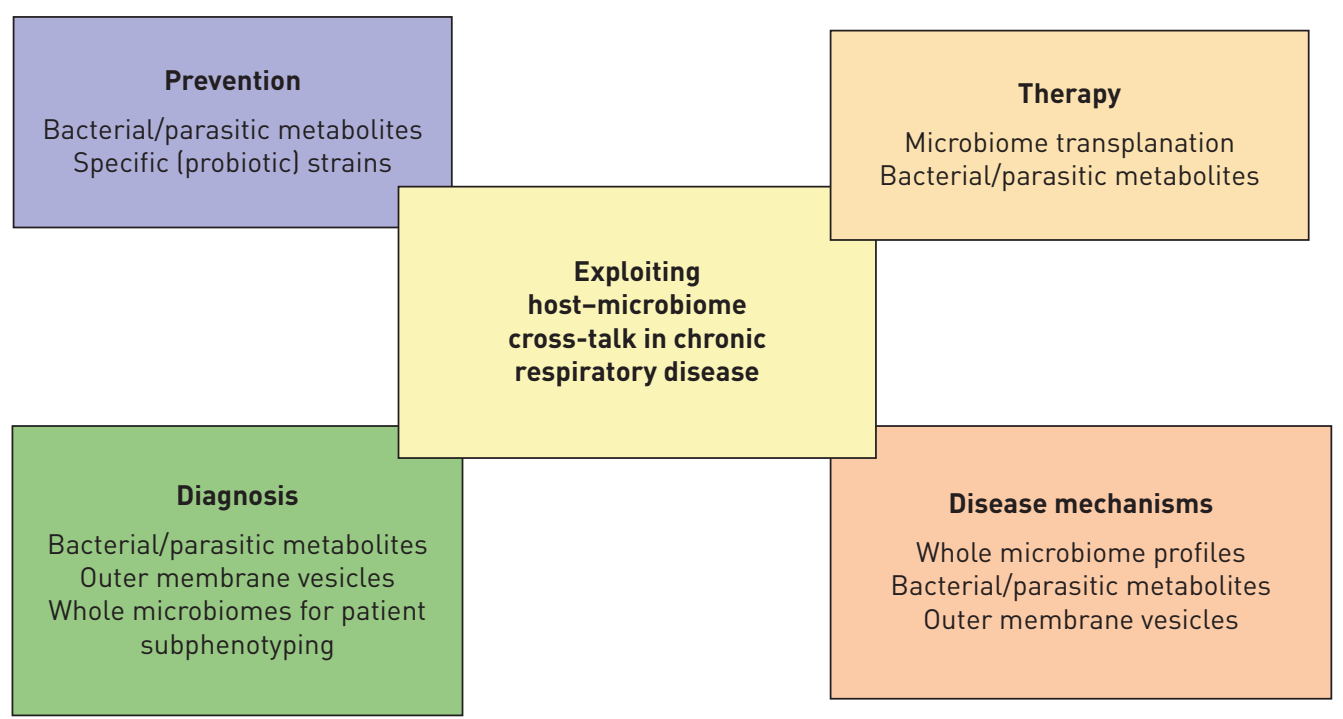

FIGURE 2 Opportunities for exploiting the host-microbiome cross-talk in chronic respiratory disease. The complexity of the host-microbe cross-talk in the lung microenvironment should be taken into account when studying chronic lung diseases. Understanding the detailed mechanisms of this cross-talk could potentially lead to the development of novel therapies, preventative strategies or superior diagnostic tools to differentiate subphenotypes of patients. 
seems encouraging; however, there remains an urgent need to better understand the complex hostmicrobiome cross-talk. There is still little information regarding the cause-and-effect relationship between the airway microbiota and disease onset/progression, except for some initial hints. Host genetics, transcriptomics and metabolomics will help resolve these issues, and provide new paradigms of how this contributes to disease severity and exacerbations. While existing treatments affect the microbiome, possibly influencing their effectiveness, the safety, optimal time point and dose of effective interventions based on whole microbiomes, metabolites or combinations thereof need to be elucidated in (longitudinal) multicentre trials. Furthermore, the microbiome could be a good indicator of patient subphenotypes and therapy responses, which should be further investigated in clinical samples, advanced disease-relevant culture models, and representative in vivo in animal models (figure 2).

Acknowledgements: The authors thank the participants of the ERS Research Seminar "Cross-talk in the lung microenvironment - implications for understanding chronic lung diseases" (Berlin, February 2018) for the fruitful discussions leading to the development of this review. Authors are listed alphabetically, except for Reinoud Gosens, Pieter Hiemstra and Sabine Bartel.

Conflict of interest: R. Gosens reports grants from Novartis, Boehringer Ingelheim, Aquilo and Chiesi, outside the submitted work. P.S. Hiemstra reports grants from Boehringer Ingelheim and Galapagos N.V., outside the submitted work. I.M. Adcock reports personal fees for lectures from AstraZeneca and Sunovion, grants from Boehringer Ingelheim, outside the submitted work. K.R. Bracke has nothing to disclose. R.P. Dickson has nothing to disclose. P.M. Hansbro has nothing to disclose. S. Krauss-Etschmann has nothing to disclose. H.H. Smits has nothing to disclose. F.R.M. Stassen has nothing to disclose. S. Bartel reports grants and personal fees for advisory board work from Bencard Allergie $\mathrm{GmbH}$, outside the submitted work.

Support statement: P.M. Hansbro is funded by a Fellowship and grants from the National Health and Medical Research Council (\#1079187, 1059238), the Rainbow Foundation and the University of Technology Sydney. S. Krauss-Etschmann is a member of the Leibniz Competition 2016 "The Lung Microbiota at the Interface between Airway Epithelium and Environment" and the Leibniz Science Campus "Evolung". H.H. Smits is supported by a ZonMW-VIDI grant (91714352) from The Netherlands Organisation for Scientific Research, and both H.H. Smits and P.S. Hiemstra are supported by a consortium grant (\#5115015) from the Netherlands Lung Foundation (NLF) and by a grant (\#12017001) from the NLF Accelerate Programme. R. Gosens and P.S. Hiemstra are supported by a consortium grant (6114009) from the NLF. Funding information for this article has been deposited with the Crossref Funder Registry.

\section{References}

1 Dickson RP, Erb-Downward JR, Martinez FJ, et al. The microbiome and the respiratory tract. Annu Rev Physiol 2016; 78: 481-504.

2 Hilty M, Burke C, Pedro H, et al. Disordered microbial communities in asthmatic airways. PLoS One 2010; 5: e8578.

3 Dickson RP, Erb-Downward JR, Freeman CM, et al. Bacterial topography of the healthy human lower respiratory tract. mBio 2017; 8: e02287-16.

4 Dickson RP, Martinez FJ, Huffnagle GB. The role of the microbiome in exacerbations of chronic lung diseases. Lancet 2014; 384: 691-702.

5 Molyneaux PL, Cox MJ, Willis-Owen SAG, et al. The role of bacteria in the pathogenesis and progression of idiopathic pulmonary fibrosis. Am J Respir Crit Care Med 2014; 190: 906-913.

6 Rogers GB, Zain NMM, Bruce KD, et al. A novel microbiota stratification system predicts future exacerbations in bronchiectasis. Ann Am Thorac Soc 2014; 11: 496-503.

7 Dickson RP, Erb-Downward JR, Falkowski NR, et al. The lung microbiota of healthy mice are highly variable, cluster by environment, and reflect variation in baseline lung innate immunity. Am J Respir Crit Care Med 2018; 198: 497-508.

8 Segal LN, Clemente JC, Tsay J-CJ, et al. Enrichment of the lung microbiome with oral taxa is associated with lung inflammation of a Th17 phenotype. Nat Microbiol 2016; 1: 16031.

9 Budden K, Shukla S, Rehman S, et al. Functional effects of microbiota in chronic respiratory disease. Lancet Respir Med 2019; 7: 907-920.

10 Durack J, Lynch SV, Nariya S, et al. Features of the bronchial bacterial microbiome associated with atopy, asthma, and responsiveness to inhaled corticosteroid treatment. J Allergy Clin Immunol 2017; 140: 63-75.

11 Sze MA, Dimitriu PA, Hayashi S, et al. The lung tissue microbiome in chronic obstructive pulmonary disease. Am J Respir Crit Care Med 2012; 185: 1073-1080.

12 Quinn RA, Adem S, Mills RH, et al. Neutrophilic proteolysis in the cystic fibrosis lung correlates with a pathogenic microbiome. Microbiome 2019; 7: 23.

13 Huang Y, Ma S-F, Espindola MS, et al. Microbes are associated with host innate immune response in idiopathic pulmonary fibrosis. Am J Respir Crit Care Med 2017; 196: 208-219.

14 Tsay J-CJ, Wu BG, Badri MH, et al. Airway microbiota is associated with upregulation of the PI3K pathway in lung cancer. Am J Respir Crit Care Med 2018; 198: 1188-1198.

15 Shukla SD, Budden KF, Neal R, et al. Microbiome effects on immunity, health and disease in the lung. Clin Transl Immunology 2017; 6: e133.

16 Marsland BJ, Gollwitzer ES. Host-microorganism interactions in lung diseases. Nat Rev Immunol 2014; 14: 827-835.

17 Hakansson AP, Orihuela CJ, Bogaert D. Bacterial-host interactions: physiology and pathophysiology of respiratory infection. Physiol Rev 2018; 98: 781-811.

18 Hiemstra PS, McCray PB, Bals R. The innate immune function of airway epithelial cells in inflammatory lung disease. Eur Respir J 2015; 45: 1150-1162. 
19 Aghapour M, Raee P, Moghaddam SJ, et al. Airway epithelial barrier dysfunction in chronic obstructive pulmonary disease: role of cigarette smoke exposure. Am J Respir Cell Mol Biol 2018; 58: 157-169.

Whitsett JA, Alenghat T. Respiratory epithelial cells orchestrate pulmonary innate immunity. Nat Immunol 2015 16: 27-35.

21 Hallstrand TS, Hackett TL, Altemeier WA, et al. Airway epithelial regulation of pulmonary immune homeostasis and inflammation. Clin Immunol 2014; 151: 1-15.

22 Rogers GB, Taylor SL, Hoffman LR, et al. The impact of CFTR modulator therapies on CF airway microbiology. J Cyst Fibros 2020; in press [https://doi.org/10.1016/j.jcf.2019.07.008].

23 Smits HH, Hiemstra PS, Prazeres da Costa C, et al. Microbes and asthma: opportunities for intervention. J Allergy Clin Immunol 2016; 137: 690-697.

24 Haspeslagh E, Heyndrickx I, Hammad H, et al. The hygiene hypothesis: immunological mechanisms of airway tolerance. Curr Opin Immunol 2018; 54: 102-108.

25 van 't Wout EF, Hiemstra PS, Marciniak SJ. The integrated stress response in lung disease. Am J Respir Cell Mol Biol 2014; 50: 1005-1009.

26 Belkaid Y, Harrison OJ. Homeostatic immunity and the microbiota. Immunity 2017; 46: 562-576.

27 van der Vlugt LEPM, Eger K, Müller C, et al. Farm dust reduces viral load in human bronchial epithelial cells by increasing barrier function and antiviral responses. J Allergy Clin Immunol 2018; 141: 1949-1952.

28 Pickering J, Teo TH, Thornton RB, et al. Bacillus licheniformis in geogenic dust induces inflammation in respiratory epithelium. Environ Res 2018; 164: 248-254.

29 Aagaard K, Ma J, Antony KM, et al. The placenta harbors a unique microbiome. Sci Transl Med 2014; 6: 237 ra65.

30 Lauder AP, Roche AM, Sherrill-Mix S, et al. Comparison of placenta samples with contamination controls does not provide evidence for a distinct placenta microbiota. Microbiome 2016; 4: 29.

31 Gollwitzer ES, Saglani S, Trompette A, et al. Lung microbiota promotes tolerance to allergens in neonates via PD-L1. Nat Med 2014; 20: 642-647.

32 Bosch AATM, de Steenhuijsen Piters WAA, van Houten MA, et al. Maturation of the infant respiratory microbiota, environmental drivers, and health consequences. A prospective cohort study. Am J Respir Crit Care Med 2017; 196: 1582-1590.

33 Man WH, van Houten MA, Mérelle ME, et al. Bacterial and viral respiratory tract microbiota and host characteristics in children with lower respiratory tract infections: a matched case-control study. Lancet Respir Med 2019; 7: 417-426.

34 Stein MM, Hrusch CL, Gozdz J, et al. Innate immunity and asthma risk in Amish and Hutterite farm children. N Engl J Med 2016; 375: 411-421.

35 von Mutius E. The microbial environment and its influence on asthma prevention in early life. J Allergy Clin Immunol 2016; 137: 680-689.

36 Dickson RP, Erb-Downward JR, Huffnagle GB. Homeostasis and its disruption in the lung microbiome. Am J Physiol Lung Cell Mol Physiol 2015; 309: L1047-L1055.

37 Venkataraman A, Bassis CM, Beck JM, et al. Application of a neutral community model to assess structuring of the human lung microbiome. mBio 2015; 6: e02284-14.

38 Kish L, Hotte N, Kaplan GG, et al. Environmental particulate matter induces murine intestinal inflammatory responses and alters the gut microbiome. PLoS One 2013; 8: e62220.

39 Hirota JA, Gold MJ, Hiebert PR, et al. The nucleotide-binding domain, leucine-rich repeat protein 3 inflammasome/IL-1 receptor I axis mediates innate, but not adaptive, immune responses after exposure to particulate matter under $10 \mu \mathrm{m}$. Am J Respir Cell Mol Biol 2015; 52: 96-105.

40 Chotirmall SH, Gellatly SL, Budden KF, et al. Microbiomes in respiratory health and disease: an Asia-Pacific perspective. Respirology 2017; 22: 240-250.

41 Biedermann L, Brülisauer K, Zeitz J, et al. Smoking cessation alters intestinal microbiota: insights from quantitative investigations on human fecal samples using FISH. Inflamm Bowel Dis 2014; 20: 1496-1501.

42 Leung JM, Tiew PY, Mac Aogáin M, et al. The role of acute and chronic respiratory colonization and infections in the pathogenesis of COPD. Respirology 2017; 22: 634-650.

43 Amatngalim GD, Schrumpf JA, Dishchekenian F, et al. Aberrant epithelial differentiation by cigarette smoke dysregulates respiratory host defence. Eur Respir J 2018; 51: 1701009.

44 Simpson JL, Baines KJ, Horvat JC, et al. COPD is characterized by increased detection of Haemophilus influenzae, Streptococcus pneumoniae and a deficiency of Bacillus species. Respirology 2016; 21: 697-704.

45 Morris A, Beck JM, Schloss PD, et al. Comparison of the respiratory microbiome in healthy nonsmokers and smokers. Am J Respir Crit Care Med 2013; 187: 1067-1075.

46 David LA, Maurice CF, Carmody RN, et al. Diet rapidly and reproducibly alters the human gut microbiome. Nature 2014; 505: 559-563.

47 Trompette A, Gollwitzer ES, Yadava K, et al. Gut microbiota metabolism of dietary fiber influences allergic airway disease and hematopoiesis. Nat Med 2014; 20: 159-166.

48 Sonnenburg ED, Smits SA, Tikhonov M, et al. Diet-induced extinctions in the gut microbiota compound over generations. Nature 2016; 529: 212-215.

49 Thorburn AN, McKenzie CI, Shen S, et al. Evidence that asthma is a developmental origin disease influenced by maternal diet and bacterial metabolites. Nat Commun 2015; 6: 7320.

50 Caesar R, Tremaroli V, Kovatcheva-Datchary P, et al. Crosstalk between gut microbiota and dietary lipids aggravates WAT inflammation through TLR signaling. Cell Metab 2015; 22: 658-668.

51 Qiu M, Huang K, Liu Y, et al. Modulation of intestinal microbiota by glycyrrhizic acid prevents high-fat diet-enhanced pre-metastatic niche formation and metastasis. Mucosal Immunol 2019; 12: 945-957.

52 Wylie KM. The virome of the human respiratory tract. Clin Chest Med 2017; 38: 11-19.

53 Planet PJ, Parker D, Cohen TS, et al. Lambda interferon restructures the nasal microbiome and increases susceptibility to Staphylococcus aureus superinfection. mBio 2016; 7: e01939-15.

54 Wark PAB, Grissell T, Davies B, et al. Diversity in the bronchial epithelial cell response to infection with different rhinovirus strains. Respirology 2009; 14: 180-186. 
Winther B, Gwaltney JM, Hendley JO. Respiratory virus infection of monolayer cultures of human nasal epithelial cells. Am Rev Respir Dis 1990; 141: 839-845.

Tarabichi $\mathrm{Y}, \mathrm{Li} \mathrm{K}, \mathrm{Hu}$ S, et al. The administration of intranasal live attenuated influenza vaccine induces changes in the nasal microbiota and nasal epithelium gene expression profiles. Microbiome 2015; 3: 74.

Salk HM, Simon WL, Lambert ND, et al. Taxa of the nasal microbiome are associated with influenza-specific IgA response to live attenuated influenza vaccine. PLoS One 2016; 11: e0162803.

Kunst H, Wickremasinghe M, Wells A, et al. Nontuberculous mycobacterial disease and Aspergillus-related lung disease in bronchiectasis. Eur Respir J 2006; 28: 352-357.

De Baets K, Dentzien-Dias P, Upeniece I, et al. Constraining the deep origin of parasitic flatworms and host-interactions with fossil evidence. Adv Parasitol 2015; 90: 93-135.

Rolff J. Why did the acquired immune system of vertebrates evolve? Dev Comp Immunol 2007; 31: 476-482.

Maizels RM, McSorley HJ. Regulation of the host immune system by helminth parasites. J Allergy Clin Immunol 2016; 138: 666-675.

McSorley HJ, Chayé MAM, Smits HH. Worms: pernicious parasites or allies against allergies? Parasite Immunol 2019; 41: e12574.

McSorley HJ, Blair NF, Smith KA, et al. Blockade of IL-33 release and suppression of type 2 innate lymphoid cell responses by helminth secreted products in airway allergy. Mucosal Immunol 2014; 7: 1068-1078.

Kang S, Shields AR, Jupatanakul N, et al. Suppressing dengue-2 infection by chemical inhibition of Aedes aegypti host factors. PLoS Negl Trop Dis 2014; 8: e3084.

Wilson MS, Taylor MD, Balic A, et al. Suppression of allergic airway inflammation by helminth-induced regulatory T cells. J Exp Med 2005; 202: 1199-1212.

van der Vlugt LEPM, Labuda LA, Ozir-Fazalalikhan A, et al. Schistosomes induce regulatory features in human and mouse CD1d(hi) B cells: inhibition of allergic inflammation by IL-10 and regulatory T cells. PLoS One 2012; 7: e30883.

Amu S, Saunders SP, Kronenberg M, et al. Regulatory B cells prevent and reverse allergic airway inflammation via FoxP3-positive T regulatory cells in a murine model. J Allergy Clin Immunol 2010; 125: 1114-1124.

Schnoeller C, Rausch S, Pillai S, et al. A helminth immunomodulator reduces allergic and inflammatory responses by induction of IL-10-producing macrophages. J Immunol 2008; 180: 4265-4272.

Zaiss MM, Rapin A, Lebon L, et al. The intestinal microbiota contributes to the ability of helminths to modulate allergic inflammation. Immunity 2015; 43: 998-1010.

Freestone PP, Hirst RA, Sandrini SM, et al. Pseudomonas aeruginosa-catecholamine inotrope interactions: a contributory factor in the development of ventilator-associated pneumonia?. Chest 2012; 142: 1200-1210.

Dickson RP, Erb-Downward JR, Prescott HC, et al. Intraalveolar catecholamines and the human lung microbiome. Am J Respir Crit Care Med 2015; 192: 257-259.

Dickson RP. The microbiome and critical illness. Lancet Respir Med 2016; 4: 59-72.

Starkey MR, Jarnicki AG, Essilfie A-T, et al. Murine models of infectious exacerbations of airway inflammation. Curr Opin Pharmacol 2013; 13: 337-344.

Hsu AC-Y, Starkey MR, Hanish I, et al. Targeting PI3K-p110 $\alpha$ suppresses influenza virus infection in chronic obstructive pulmonary disease. Am J Respir Crit Care Med 2015; 191: 1012-1023.

Darveaux JI, Lemanske RF Jr. Infection-related asthma. J Allergy Clin Immunol Pract 2014; 2: 658-663. asthmatics are associated with increased 8-isoprostane and airway neutrophilia. Free Radic Res 2010; 44: 146-154. King PT, Sharma R, O’Sullivan K, et al. Nontypeable Haemophilus influenzae induces sustained lung oxidative stress and protease expression. PLoS One 2015; 10: e0120371.

Tay HL, Kaiko GE, Plank M, et al. Antagonism of miR-328 increases the antimicrobial function of macrophages and neutrophils and rapid clearance of non-typeable Haemophilus influenzae (NTHi) from infected lung. PLoS Pathog 2015; 11: e1004549.

Kim RY, Horvat JC, Pinkerton JW, et al. MicroRNA-21 drives severe, steroid-insensitive experimental asthma by amplifying phosphoinositide 3-kinase-mediated suppression of histone deacetylase 2. J Allergy Clin Immunol 2017; 139: 519-532.

Kim RY, Pinkerton JW, Essilfie AT, et al. Role for NLRP3 inflammasome-mediated, IL-1 $\beta$-dependent responses in severe, steroid-resistant asthma. Am J Respir Crit Care Med 2017; 196: 283-297.

Alnahas S, Hagner S, Raifer H, et al. IL-17 and TNF- $\alpha$ are key mediators of Moraxella catarrhalis triggered exacerbation of allergic airway inflammation. Front Immunol 2017; 8: 1562.

Essilfie A-T, Horvat JC, Kim RY, et al. Macrolide therapy suppresses key features of experimental steroid-sensitive and steroid-insensitive asthma. Thorax 2015; 70: 458-467.

1199-1201.

Hansbro PM, Kim RY, Starkey MR, et al. Mechanisms and treatments for severe, steroid-resistant allergic airway disease and asthma. Immunol Rev 2017; 278: 41-62.

Taylor SL, Leong LEX, Choo JM, et al. Inflammatory phenotypes in patients with severe asthma are associated with distinct airway microbiology. J Allergy Clin Immunol 2018; 141: 94-103.

Kim B-S, Lee E, Lee M-J, et al. Different functional genes of upper airway microbiome associated with natural course of childhood asthma. Allergy 2018; 73: 644-652.

Chiu C-Y, Chan Y-L, Tsai Y-S, et al. Airway microbial diversity is inversely associated with mite-sensitized rhinitis and asthma in early childhood. Sci Rep 2017; 7: 1820.

Goleva E, Harris JK, Robertson CE, et al. Airway microbiome and responses to corticosteroids in corticosteroid-resistant asthma patients treated with acid suppression medications. J Allergy Clin Immunol 2017; 140: $860-862$.

Cuthbertson L, Rogers GB, Walker AW, et al. Respiratory microbiota resistance and resilience to pulmonary exacerbation and subsequent antimicrobial intervention. ISME J 2016; 10: 1081-1091.

Cox MJ, Turek EM, Hennessy C, et al. Longitudinal assessment of sputum microbiome by sequencing of the $16 \mathrm{~S}$ rRNA gene in non-cystic fibrosis bronchiectasis patients. PLoS One 2017; 12: e0170622. 

endomembrane and HLA pathways modifying cystic fibrosis pulmonary phenotypes. Am J Hum Genet 2015; 96: 318-328.

92 Heijink IH, Nawijn MC, Hackett T-L. Airway epithelial barrier function regulates the pathogenesis of allergic asthma. Clin Exp Allergy 2014; 44: 620-630.

93 Wark PAB, Johnston SL, Bucchieri F, et al. Asthmatic bronchial epithelial cells have a deficient innate immune response to infection with rhinovirus. J Exp Med 2005; 201: 937-947.

94 Contoli M, Message SD, Laza-Stanca V, et al. Role of deficient type III interferon- $\lambda$ production in asthma exacerbations. Nat Med 2006; 12: 1023-1026.

95 Hsu AC-Y, Parsons K, Moheimani F, et al. Impaired antiviral stress granule and IFN- $\beta$ enhanceosome formation enhances susceptibility to influenza infection in chronic obstructive pulmonary disease epithelium. Am J Respir Cell Mol Biol 2016; 55: 117-127.

96 Bedke N, Sammut D, Green B, et al. Transforming growth factor-beta promotes rhinovirus replication in bronchial epithelial cells by suppressing the innate immune response. PLoS One 2012; 7: e44580.

97 Principi N, Daleno C, Esposito S. Human rhinoviruses and severe respiratory infections: is it possible to identify at-risk patients early? Expert Rev Anti Infect Ther 2014; 12: 423-430.

98 Finney LJ, Belchamber KBR, Fenwick PS, et al. Human rhinovirus impairs the innate immune response to bacteria in alveolar macrophages in chronic obstructive pulmonary disease. Am J Respir Crit Care Med 2019; 199: 1496-1507.

99 Quantius J, Schmoldt C, Vazquez-Armendariz AI, et al. Influenza virus infects epithelial stem/progenitor cells of the distal lung: impact on Fgfr2b-driven epithelial repair. PLoS Pathog 2016; 12: e1005544.

100 Russell SL, Gold MJ, Hartmann M, et al. Early life antibiotic-driven changes in microbiota enhance susceptibility to allergic asthma. EMBO Rep 2012; 13: 440-447.

101 Adami AJ, Bracken SJ, Guernsey LA, et al. Early-life antibiotics attenuate regulatory $\mathrm{T}$ cell generation and increase the severity of murine house dust mite-induced asthma. Pediatr Res 2018; 84: 426-434.

102 Jin C, Lagoudas GK, Zhao C, et al. Commensal microbiota promote lung cancer development via $\gamma \delta \mathrm{T}$ cells. Cell 2019; 176: 998-1013.

103 Keely S, Talley NJ, Hansbro PM. Pulmonary-intestinal cross-talk in mucosal inflammatory disease. Mucosal Immunol 2012; 5: 7-18.

104 Mateer SW, Mathe A, Bruce J, et al. IL-6 drives neutrophil-mediated pulmonary inflammation associated with bacteremia in murine models of colitis. Am J Pathol 2018; 188: 1625-1639.

105 Mateer SW, Maltby S, Marks E, et al. Potential mechanisms regulating pulmonary pathology in inflammatory bowel disease. J Leukoc Biol 2015; 98: 727-737.

106 Chambers DC, Gellatly SL, Hugenholtz P, et al. JTD special edition 'Hot Topics in COPD' - The microbiome in COPD. J Thorac Dis 2014; 6: 1525-1531.

107 Budden KF, Gellatly SL, Wood DL, et al. Emerging pathogenic links between microbiota and the gut-lung axis. Nat Rev Microbiol 2017; 15: 55-63.

108 Beckett EL, Stevens RL, Jarnicki AG, et al. A new short-term mouse model of chronic obstructive pulmonary disease identifies a role for mast cell tryptase in pathogenesis. J Allergy Clin Immunol 2013; 131: 752-762.

109 Hansbro PM, Hamilton MJ, Fricker M, et al. Importance of mast cell Prss31/transmembrane tryptase/tryptase- $\gamma$ in lung function and experimental chronic obstructive pulmonary disease and colitis. J Biol Chem 2014; 289: 18214-18227.

110 Jones B, Donovan C, Liu G, et al. Animal models of COPD: what do they tell us? Respirology 2017; 22 : 21-32.

111 Fricker M, Goggins BJ, Mateer S, et al. Chronic cigarette smoke exposure induces systemic hypoxia that drives intestinal dysfunction. JCI Insight 2018; 3: 94040.

112 Clarke TB. Early innate immunity to bacterial infection in the lung is regulated systemically by the commensal microbiota via nod-like receptor ligands. Infect Immun 2014; 82: 4596-4606.

113 Sagar S, Morgan ME, Chen S, et al. Bifidobacterium breve and Lactobacillus rhamnosus treatment is as effective as budesonide at reducing inflammation in a murine model for chronic asthma. Respir Res 2014; 15: 46.

114 Arrieta M-C, Stiemsma LT, Dimitriu PA, et al. Early infancy microbial and metabolic alterations affect risk of childhood asthma. Sci Transl Med 2015; 7: 307ra152.

115 Pragman AA, Kim HB, Reilly CS, et al. The lung microbiome in moderate and severe chronic obstructive pulmonary disease. PLoS One 2012; 7: e47305.

116 Rosen R, Hu L, Amirault J, et al. 16S community profiling identifies proton pump inhibitor related differences in gastric, lung, and oropharyngeal microflora. J Pediatr 2015; 166: 917-923.

117 Singanayagam A, Glanville N, Cuthbertson L, et al. Inhaled corticosteroid suppression of cathelicidin drives dysbiosis and bacterial infection in chronic obstructive pulmonary disease. Sci Transl Med 2019; 11: eaav3879.

118 Gulmez SE, Holm A, Frederiksen H, et al. Use of proton pump inhibitors and the risk of community-acquired pneumonia: a population-based case-control study. Arch Intern Med 2007; 167: 950-955.

119 Singh S, Amin AV, Loke YK. Long-term use of inhaled corticosteroids and the risk of pneumonia in chronic obstructive pulmonary disease: a meta-analysis. Arch Intern Med 2009; 169: 219-229.

120 Simpson JL, Powell H, Baines KJ, et al. The effect of azithromycin in adults with stable neutrophilic COPD: a double blind randomised, placebo controlled trial. PLoS One 2014; 9: e105609.

121 Sharma G, Im SH. Probiotics as a potential immunomodulating pharmabiotics in allergic diseases: current status and future prospects. Allergy Asthma Immunol Res 2018; 10: 575-590.

122 Round JL, Mazmanian SK. The gut microbiota shapes intestinal immune responses during health and disease. Nat Rev Immunol 2009; 9: 313-323.

123 Britton RA, Young VB. Role of the intestinal microbiota in resistance to colonization by Clostridium difficile. Gastroenterology 2014; 146: 1547-1553.

124 Lawley TD, Clare S, Walker AW, et al. Targeted restoration of the intestinal microbiota with a simple, defined bacteriotherapy resolves relapsing Clostridium difficile disease in mice. PLoS Pathog 2012; 8: e1002995.

125 Petrof EO, Gloor GB, Vanner SJ, et al. Stool substitute transplant therapy for the eradication of Clostridium difficile infection: "rePOOPulating" the gut. Microbiome 2013; 1: 3. 


$$
\text { breve }
$$
responses. Eur Respir J 2015; 46: Suppl. 59, PA5089.
Case RJ, Labbate M, Kjelleberg S. AHL-driven quo Proteobacteria. ISME J 2008; 2: 345-349.

133 Losa D, Köhler T, Bacchetta M, et al. Airway epithelial cell integrity protects from cytotoxicity of Pseudomonas aeruginosa quorum-sensing signals. Am J Respir Cell Mol Biol 2015; 53: 265-275.

134 Lee RJ, Xiong G, Kofonow JM, et al. T2R38 taste receptor polymorphisms underlie susceptibility to upper respiratory infection. J Clin Invest 2012; 122: 4145-4159.

135 Maurer S, Wabnitz GH, Kahle NA, et al. Tasting Pseudomonas aeruginosa biofilms: human neutrophils express the bitter receptor T2R38 as sensor for the quorum sensing molecule N-(3-oxododecanoyl)-L-homoserine lactone. Front Immunol 2015; 6: 369.

136 Jaggupilli A, Singh N, De JV, et al. Characterization of the binding sites for bacterial acyl homoserine lactones (AHLs) on human bitter taste receptors (T2Rs). ACS Infect Dis 2018; 4: 1146-1156.

137 Gaida MM, Dapunt U, Hänsch GM. Sensing developing biofilms: the bitter receptor T2R38 on myeloid cells. Pathog Dis 2016; 74: ftw004.

138 Zimmermann S, Wagner C, Müller W, et al. Induction of neutrophil chemotaxis by the quorum-sensing molecule N-(3-oxododecanoyl)-L-homoserine lactone. Infect Immun 2006; 74: 5687-5692.

139 Kaparakis-Liaskos M, Ferrero RL. Immune modulation by bacterial outer membrane vesicles. Nat Rev Immunol 2015; 15: 375-387.

140 Brown L, Wolf JM, Prados-Rosales R, et al. Through the wall: extracellular vesicles in Gram-positive bacteria, mycobacteria and fungi. Nat Rev Microbiol 2015; 13: 620-630.

141 Schwechheimer C, Kuehn MJ. Outer-membrane vesicles from Gram-negative bacteria: biogenesis and functions. Nat Rev Microbiol 2015; 13: 605-619.

142 Volgers C, Savelkoul PHM, Stassen FRM. Gram-negative bacterial membrane vesicle release in response to the host-environment: different threats, same trick? Crit Rev Microbiol 2018; 44: 258-273.

143 Sharpe SW, Kuehn MJ, Mason KM. Elicitation of epithelial cell-derived immune effectors by outer membrane vesicles of nontypeable Haemophilus influenzae. Infect Immun 2011; 79: 4361-4369.

144 Tan TT, Morgelin M, Forsgren A, et al. Haemophilus influenzae survival during complement-mediated attacks is promoted by Moraxella catarrhalis outer membrane vesicles. J Infect Dis 2007; 195: 1661-1670.

145 Vidakovics ML, Jendholm J, Mörgelin M, et al. B cell activation by outer membrane vesicles - a novel virulence mechanism. PLoS Pathog 2010; 6: e1000724.

146 Mashburn LM, Whiteley M. Membrane vesicles traffic signals and facilitate group activities in a prokaryote. Nature 2005; 437: 422-425.

147 Lin J, Zhang W, Cheng J, et al. A Pseudomonas T6SS effector recruits PQS-containing outer membrane vesicles for iron acquisition. Nat Commun 2017; 8: 14888.

148 Volgers C, Benedikter BJ, Grauls GE, et al. Immunomodulatory role for membrane vesicles released by THP-1 macrophages and respiratory pathogens during macrophage infection. BMC Microbiol 2017; 17: 216.

149 Jung AL, Stoiber C, Herkt CE, et al. Legionella pneumophila-derived outer membrane vesicles promote bacterial replication in macrophages. PLoS Pathog 2016; 12: e1005592.

150 Park K-S, Lee J, Jang SC, et al. Pulmonary inflammation induced by bacteria-free outer membrane vesicles from Pseudomonas aeruginosa. Am J Respir Cell Mol Biol 2013; 49: 637-645.

151 Schulz C, Lai X, Bertrams W, et al. THP-1-derived macrophages render lung epithelial cells hypo-responsive to Legionella pneumophila - a systems biology study. Sci Rep 2017; 7: 11988.

152 Jatzlauk G, Bartel S, Heine H, et al. Influences of environmental bacteria and their metabolites on allergies, asthma, and host microbiota. Allergy 2017; 72: 1859-1867.

153 Gerhold K, Blümchen K, Bock A, et al. Endotoxins prevent murine IgE production, $\mathrm{T}_{\mathrm{H}} 2$ immune responses, and development of airway eosinophilia but not airway hyperreactivity. J Allergy Clin Immunol 2002; 110: 110-116.

154 Mazmanian SK, Cui HL, Tzianabos AO, et al. An immunomodulatory molecule of symbiotic bacteria directs maturation of the host immune system. Cell 2005; 122: 107-118.

155 Kitagaki K, Businga TR, Kline JN. Oral administration of CpG-ODNs suppresses antigen-induced asthma in mice. Clin Exp Immunol 2006; 143: 249-259.

156 Shim JU, Lee SE, Hwang W, et al. Flagellin suppresses experimental asthma by generating regulatory dendritic cells and T cells. J Allergy Clin Immunol 2016; 137: 426-435.

157 Kepert I, Fonseca J, Müller C, et al. D-tryptophan from probiotic bacteria influences the gut microbiome and allergic airway disease. J Allergy Clin Immunol 2017; 139: 1525-1535.

158 Zhou S, Huang Y, Liang B, et al. Systemic and mucosal pre-administration of recombinant Helicobacter pylori neutrophil-activating protein prevents ovalbumin-induced allergic asthma in mice. FEMS Microbiol Lett 2017; 364: fnw288.

159 Rzepecka J, Coates ML, Saggar M, et al. Small molecule analogues of the immunomodulatory parasitic helminth product ES-62 have anti-allergy properties. Int J Parasitol 2014; 44: 669-674.

160 Daniłowicz-Luebert E, Steinfelder S, Kühl AA, et al. A nematode immunomodulator suppresses grass pollen-specific allergic responses by controlling excessive Th2 inflammation. Int J Parasitol 2013; 43: 201-210. 
161 Filbey KJ, Camberis $\mathrm{M}$, Chandler $\mathrm{J}$, et al. Intestinal helminth infection promotes IL-5- and $\mathrm{CD} 4^{+} \mathrm{T}$ cell-dependent immunity in the lung against migrating parasites. Mucosal Immunol 2019; 12: 352-362.

162 Johnston CJC, Smyth DJ, Kodali RB, et al. A structurally distinct TGF- $\beta$ mimic from an intestinal helminth parasite potently induces regulatory T cells. Nat Commun 2017; 8: 1741.

163 Everts B, Hussaarts L, Driessen NN, et al. Schistosome-derived omega-1 drives Th2 polarization by suppressing protein synthesis following internalization by the mannose receptor. J Exp Med 2012; 209: 1753-1767.

164 Hisert KB, Heltshe SL, Pope C, et al. Restoring cystic fibrosis transmembrane conductance regulator function reduces airway bacteria and inflammation in people with cystic fibrosis and chronic lung infections. Am J Respir Crit Care Med 2017; 195: 1617-1628. 